\title{
Effect of Huoxuejiangu Decoction on Osteoarthritis
}

\author{
Zike He, Kaifei He, Shangzeng Wang \\ Henan Provincial Hospital orthopaedics, Zhengzhou, Henan
}

\begin{abstract}
Objective: To explore the clinical intervention effect of applying Huoxue Jiangu Decoction in the treatment of patients with osteoarthritis. Methods: Research work was carried out in our hospital from December 2018 to December 2019. A total of 100 patients with osteoarthritis received treatment during this period were selected and divided into two groups using random number method. One group was given sodium hyaluronate injection for treatment, which was the control group, and the other group was given Huoxuejiangu Decoction combined with sodium hyaluronate injection, which was the experimental group. The clinical treatment of the two groups of patients was compared and analyzed. Results: The patients' pain levels were compared. There was no significant difference between the groups before the intervention treatment. After treatment, the experimental group score was (3.05 \pm 0.55$)$, which was lower than the control group, the improvement effect was more significant, and the difference between the groups was significant $(\mathrm{P}<0.05)$, the experimental group had a better effect. The clinical treatment of the two groups of patients was compared and analyzed. There was no significant difference in the WOMAS index between the two groups before treatment. After treatment, the score of the experimental group was (34.33 \pm 6.98$)$, and the improvement rate was $(37.45 \pm 13.22)$. After treatment, the score was lower and the improvement rate was higher, which was significantly higher than the control group $(\mathrm{P}<0.05)$, which was significant. The treatment effective rate of the patients in the experimental group was $80.00 \%$, and the treatment effective rate of the patients in the control group was $64.00 \%$. When comparing, the effective rate of the experimental group was higher, and the data comparison between the groups was significantly different. Conclusion: For the patients with osteoarthritis, the application of Huoxue Jiangu Decoction has a significant clinical effect, which can reduce the patient's pain level, improve the patient's clinical symptoms, have high clinical value, and it can be promoted.
\end{abstract}

\section{1 introduction}

Osteoarthritis is relatively common in the clinic. Patients exhibit chronic degenerative lesions, which will show symptoms such as stiffness, dysfunction and joint pain. It is necessary to give patients timely and effective treatment, otherwise it will make it difficult for patients to walk normally. On this basis, it is necessary to give patients an effective treatment for arthritis, promote their recovery, and improve their quality of life [1]. This study taking patients in our hospital as an example, applying Huoxue Jiangu Decoction to explore its clinical application effect.

\section{Patient data and research methods}

\subsection{General information}

The research work was carried out in our hospital from December 2018 to December 2019. A total of 100 patients with osteoarthritis who received treatment during this period were selected and divided into two groups using random number method. One group was given sodium hyaluronate injection as control group, and the other group was given Huoxuejiangu Decoction combined with sodium hyaluronate injection as experimental group. The number of each group was 50 . There were 57 male patients and 43 female patients. The youngest is 42 years old and the oldest is 71 years old. The corresponding average age of the patients is $(62.35 \pm 2.62)$ years old. There is no significant difference in the general data of the two groups of patients, which can be compared between groups.

Inclusion criteria: Patients all met the diagnostic criteria for arthritis developed by the American College of Rheumatology: morning stiffness was more than 30 minutes, showed joint pain, and there were joint friction sounds during exercise. The patient was informed and agreed to participate in this study.

Exclusion criteria: The patient has a history of mental illness, knee deformity, the patient has a mental disorder, the patient has periostitis, and the clinical data is incomplete. 


\subsection{Research methods}

In this study, the control group was given sodium hyaluronate injection, and the corresponding sodium hyaluronate injection was injected into the patient's joint cavity with a dose of $2.5 \mathrm{ml}$. The commercial name of the sodium hyaluronate selected in this study was Bai Bei, and it is produced by Shanghai Baijiayi Pharmaceutical Co., Ltd., the corresponding production batch number is: National Pharmaceutical Standard H20000643, after which it will be injected once a week for five consecutive weeks of intervention. In the specific implementation process, help the patient to choose a sitting position, it will promote the patient's knee joint to flex, maintain the knee joint at 90 degrees to promote natural sagging, and routinely disinfect the intervention site, select the patella inferior or lateral underside for approach. The 7-gauge injection needle is applied, and the needle is inserted into the middle and upward 45 degrees, and $25 \mathrm{mg}$ of sodium hyaluronate is injected into the joint cavity without blood return.

Patients in the experimental group were treated with sodium hyaluronate injection combined with Huoxue Jiangu Decoction. The specific pharmaceutical composition is: Chuanduan, safflower, papaya, heliotrope, roasted licorice, myrrh, angelica, Qiang Huo are all 10g, white peony root, windproof are $12 \mathrm{~g}, 15 \mathrm{~g}$ of Bupleurum and Tendon. Use stainless steel or pottery to cook the medicine. The cold water needs to be $3-5 \mathrm{~cm}$ on the surface of the medicine, the head frying time is 10-25 minutes, and the second frying time needs to be controlled at 15-20 minutes. Mix and concentrate the medicine finally into $300 \mathrm{ml}$, take it twice. Take $150 \mathrm{ml}$ of mixed medicine twice a day for morning and evening, and take seven consecutive days as a course of treatment. Patients receive five consecutive courses of intervention. The injection method and dosage of sodium hyaluronate are the same as the control group.

\subsection{Observation indicators}

VAS scale: The visual analog scale is mainly used. The total pain level is 10 points, 0 point is no pain, and 10 points are severe pain. The higher the patient's score, the more severe the patient's pain.
WOMAS index: It is a patient's osteoarthritis index. It is an internationally recognized evaluation standard. It needs to observe the three indicators of the patient's joint pain, stiffness and function, and give the patient a quantitative score to calculate the patient's improvement. The full score is 100 points, and a 0 point indicates that the patient is normal, and a high score indicates that the patient has severe symptoms. To calculate the improvement rate, you need to subtract the pre-treatment score of the patient from the post-treatment score and compare it with the pre-treatment score [2].

Therapeutic effect: It mainly includes four indicators: clinical control, effective, marked effective and ineffective. Among them, clinical control means the clinical symptoms of the patient basically disappeared, marked effective means the patient's clinical symptoms and signs were alleviated, effective means that the patient's clinical symptoms had be improved, and ineffective means that the patients didn't have clinical changes. The total effective rate in this study excluded the ineffective rate.

\subsection{Statistical methods}

Using the statistical software SPSS20.0 as a tool, perform statistical analysis on the data presented in this study, verifying the $t$ value of the comparison result of the measurement data $( \pm \mathrm{s})$, and verifying the 2 value of the comparison result of the count data (n, \%), when the result shows $\mathrm{P}<0.05$, it means that the difference between the groups has statistical analysis value [3].

\section{Results}

\subsection{VAS score}

Comparing the pain level of patients, there was no significant difference between the data before intervention. After treatment, the experimental group score was $(3.05 \pm 0.55)$, which was lower than that of the control group, the improvement effect was more significant, and the data difference between the groups was significant $(\mathrm{P}<0.05)$, the experimental group had better effect.

Table 1 Comparison of VAS scores between two groups $( \pm \mathrm{s})$

\begin{tabular}{ccc}
\hline Group & Before treatment & After treatment \\
\hline Experimental group $(\mathbf{n = 5 0})$ & $8.11 \pm 0.77$ & $3.05 \pm 0.55$ \\
Control group $(\mathbf{n = 5 0})$ & $8.14 \pm 0.85$ & $4.34 \pm 0.85$ \\
$\mathrm{X}^{2}$ & 0.456 & 5.405 \\
$\mathrm{P}$ & $>0.05$ & $<0.05$
\end{tabular}

\subsection{Comparison of WOMAS index changes}

There was no significant difference in the WOMAS index between the two groups before treatment. After treatment, the experimental group was $(34.33 \pm 6.98)$, and the improvement rate was (37.45 \pm 13.22$)$. After treatment, the score was lower and the improvement rate was higher, which was significantly higher than the control group ( $\mathrm{P}$ $<0.05$ ), which is significant. 
Table 2 Comparison of changes in WOMAS index between two groups $( \pm \mathrm{s})$

\begin{tabular}{cccc}
\hline Group & Before treatment & After treatment & Improvement rate \\
\hline $\begin{array}{c}\text { Experimental group } \\
(\mathbf{n = 5 0})\end{array}$ & $56.44 \pm 8.45$ & $34.33 \pm 6.98$ & $37.45 \pm 13.22$ \\
Control group $(\mathbf{n = 5 0})$ & $57.05 \pm 8.54$ & $38.94 \pm 7.45$ & $32.04 \pm 12.33$ \\
$X^{2}$ & 0.389 & 3.024 & 4.034 \\
$P$ & $>0.05$ & $<0.05$ & $<0.05$
\end{tabular}

\subsection{Treatment effect}

The treatment effective rate of the patients in the experimental group was $80.00 \%$, and the treatment

Table 3 Comparison of treatment effect between the two groups [ $(\%)$ ] effective rate of the patients in the control group was $64.00 \%$. When comparing, the effective rate of the experimental group was higher, and the data comparison between the groups was significantly different.

\begin{tabular}{|c|c|c|c|c|c|}
\hline Group & Clinical control & $\begin{array}{l}\text { Marked } \\
\text { effective }\end{array}$ & Effective & Ineffective & Effective rate \\
\hline $\begin{array}{l}\text { Experimental } \\
\text { group }(n=50)\end{array}$ & $20(40.00)$ & $11(22.00)$ & $9(18.00)$ & $10(2.00)$ & $40(80.00)$ \\
\hline $\begin{array}{c}\text { Control group } \\
\quad(\mathbf{n}=\mathbf{5 0})\end{array}$ & $11(22.00)$ & $12(24.00)$ & $9(18.00)$ & $18(36.00)$ & $32(64.00)$ \\
\hline $\mathbf{X} 2$ & & & & & 12.856 \\
\hline $\mathbf{P}$ & & & & & $<0.05$ \\
\hline
\end{tabular}

\section{Discussion}

Osteoarthritis is a chronic degenerative disease. Patients will show joint pain, stiffness and other conditions, which have a greater impact on the patient's quality of daily life and even affect the patient's walking function. Starting from the perspective of traditional Chinese medicine and analyzing this condition, osteoarthritis belongs to a category of "bone paralysis" and "muscle paralysis". From the clinical development point of view, osteoarthritis patients mainly exhibit joint pain, which has a relatively large impact on the patient's physical and mental health. The patient's pain symptoms are mainly painful and not coherent, or coherent and not painful. Therefore, clinical treatment is given to patients in the process by relieving pain from pain. For osteoarthritis, its pathogenesis is mainly affected by biological factors and mechanical factors, which lead to the synthesis of the articular cartilage system, external cell matrix and subchondral bone of the patient, thereby reducing the patient's balance [4]. At the same time, the occurrence of this disease may be related to the patient's aging, obesity, metabolic disorders and other factors. The patient will show changes in chondrocyte and matrix morphology, thereby improving the patient's molecular biology, biomechanics, biochemistry and other related changes, resulting in patients with reduced cartilage, softening, ulcers, fibrosis and other symptoms [5].

In the actual development of the work, Huoxue Jiangu Decoction is applied. Among them, Achyranthes bidentata and bone crushing have the effect of strengthening muscles and bones, and nourishing liver and kidney. At the same time, it can promote the health of the waist and knees of the patient and give the patient the effect of promoting blood circulation and analgesic. Walnut, safflower, etc. in the actual application process, has the application effect of promoting blood circulation and removing blood stasis, through menstruation and analgesia, of which Angelica can promote the function of promoting blood circulation and blood, to achieve the effect of removing blood stasis. When applying Clematis, it can achieve the effect of removing wind and dehumidification, and Activating meridians and channels[6]. The application of Chuanqiong has the effect of promoting blood circulation and qi, and when it is applied, it can relieve wind and pain. The Coprinus chinensis has a certain blood circulation effect and promotes meridians and collaterals. When this prescription is applied, it can achieve the functions of promoting blood circulation and removing blood stasis, strengthening kidney and bone, and relieving pain through menstruation. In other words, in the process of treating osteoarthritis, the application of Huoxue Jiangu Decoction can effectively improve the clinical symptoms of the patient, reduce the patient's pain, relieve the patient's joint stiffness, and reduce the symptom problems caused by the disease. After the application of this method in this study, the results showed that: the patient's pain level was compared, there was no significant difference between the groups before intervention, after treatment, the experimental group score was $(3.05 \pm 0.55)$, which is lower than the control group. The improvement effect was more significant, the data difference between the groups was significant $(\mathrm{P}<0.05)$, and the experimental group had a better effect. There was no significant difference in the WOMAS index between the two groups before treatment. After treatment, the index of the experimental group was $(34.33 \pm 6.98)$, and the improvement rate was 
(37.45 \pm 13.22$)$. After treatment, the score was lower and the improvement rate was higher, which was significantly higher than the control group $(\mathrm{P}<0.05)$. The treatment effective rate of the patients in the experimental group was $80.00 \%$, and the treatment effective rate of the patients in the control group was $64.00 \%$. When comparing, the effective rate of the experimental group was higher, and the data comparison between the groups was significantly different. It can be seen from the results that the corresponding clinical effect is very significant in the application of Huoxue Jiangu Decoction in the treatment of osteoarthritis, which can eliminate the patient's pain, stiffness and dysfunction problems, the patient's clinical symptoms were also alleviated [7].

In summary, for the patients with osteoarthritis, the treatment method and application of Huoxue Jiangu Decoction have a significant clinical effect, which can reduce the patient's pain level, improve the patient's clinical symptoms, and have higher clinical value and can be promoted.

\section{Acknowledgement}

Here, I would like to thank my tutor and my teacher who helped me, and wish them good health and smooth work. At the same time, I also pay a high tribute to the researchers of the relevant literature, and your research has provided me with great reference value.

\section{References:}

1. Li Qianyi, “Analysis of the effect of Huoxue Jiangu Decoction on osteoarthritis[J]" in Doctor, 2017, 2(07): 69-70.

2. Tian He, "Clinical study of Qiangshen Jiangu Decoction in treating knee osteoarthritis (deficiency of liver and kidney) [D]" in Changchun University of Traditional Chinese Medicine, 2015.

3. Luo Wanhua, "Observation on the clinical effect of Qianjin Jiangu Decoction on knee osteoarthritis[D]" in Shandong University of Traditional Chinese Medicine, 2011.

4. Shao Songtao, "Clinical Study on Bushen Jiangu Decotion Combined with Bone Peptide Injection in Treatment of Knee Osteoarthritis $[\mathrm{J}]$ " in Practical Clinical Journal of Integrated Traditional Chinese and Western Medicine,2019,19(05):76-78.

5. Guo Lubin, "Jiangu Decoction combined with staged rehabilitation training for knee osteoarthritis[J]" in Jilin Journal of Traditional Chinese Medicine,2019,39(07):897-900.

6. Wang Yuankai, "Clinical study of Huoxuejiangu Decoction combined with decompression bone grafting to treat non-traumatic femoral head necrosis [D]" in Heilongjiang University of Traditional Chinese Medicine, 2006.

7. Qin Xingyun, Wang Yi, Liu Lihua, Mao Hongling, Wu Fangxia, "Chushi Jiangu Decoction for the
Treatment of 49 Cases of Knee Osteoarthritis[J]" in Gansu Journal of Traditional Chinese Medicine,2009,22(11):18-19. 